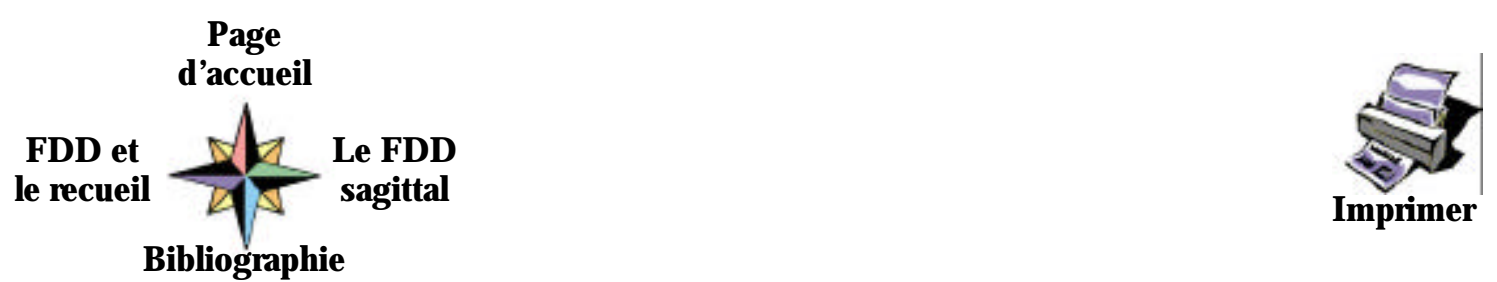

\title{
Quelques remarques sur l'utilisation des modèles
}

Comme il s'agit ici avant tout de foumir un point de départ à la discussion, je me contenterai de donner quelques éléments de définition et de commenter rapidement un schéma sur le fonctionnement des modèles.

\section{I - Définitions}

Les acceptions du terme modèle sont multiples, mais il n'est pas certain qu'on ait avantage à trop hésiter. Il semble que Haggett ait bien clanifié le débat, quand il écrit : " On utilise (le terme de modèle) comme un nom en impliquant une représentation, comme un adjectif en impliquant un degré de perfection, comme un verbe en impliquant une démonstration ou le désir de montrer à quoi ressemble une chose ". " 0 n peut donc définir le modèle comme une représentation idéalisée de la réalité élaborée en vue d'une démonstration " (Models in geography, p. 22-23). Ceci est le cour de la définition, son point central. Il existe un certain nombre de connotations fréquentes, mais pas obligatoires. N ous y reviendrons, mais pour le moment, il semble utile de situer la notion de modèle en face de quelques autres notions, assez proches, et parfois confondues avec le modèle, implicitement ou explicitement.

La notion de concept d'abord. Comme le modèle, il est une abstraction à partir de la réalité, légitime et nécessaire de la même façon, à condition évidemment qu'on se souvienne dans les deux cas que la représentation n'est pas le représenté. Mais le concept est purement mental, et ne s'exprime que par des mots. De plus, il lui manque l'aspect dynamique que l'on trouve souvent dans le modèle. Le "crêt ", forme de relief, est un concept ; il implique, dans sa définition classique, un modèle de genèse, mais le concept et le modèle, bien que liés, ne coïncident pas.

La notion de théorie ensuite. Il semblerait utile de la distinguer du modèle, ce qu'on ne fait pas toujours. La théorie qu'un vocabulaire de la philosophie définit comme une " construction intellectuelle qui essaie de relier le plus grand nombre de phénomènes observés et de lois particulières en un ensemble cohérent commandé par un principe général explicatif du tout envisagé ", serait nettement plus complète et complexe que le modèle. Le modèle senvirait à saisir une part plus limitée du réel, sans impliquer de principes généraux. Il y aurait d'ailleurs alors une hiérarchie de certitude décroissante et de généralité croissante, en passant de la loi au modèle, puis du modèle à la théorie.

Mais il y a des rapports entre modèle et théorie. Une théorie peut englober un ou plusieurs modèles, qu'elle situe dans un cadre plus général, qui peut aller jusqu'à constituer une conception du monde ou même une idéologie. A. Régnier a bien montré que certains modèles de la physique sont étroitement liés à des théories, dans la mesure où ils parlent en quelque sorte le même langage qu'elles. 
Dans cette perspective, beaucoup de "théories" de la géographie sont en fait des modèles. Par exemple, il y a un modèle de la pénéplanation selon Davis, qui fait peutêtre référence à une théorie, celle de l'actualisme, mais n'en est pas une au sens plein du terme.

Avec son goût du concret, et sa méfiance devant les références générales explicites, on peut penser que la géographie élabore beaucoup de modèles et peu de théories. Mais elle fait souvent référence à des théories élaborées par ailleurs, dans d'autres disciplines, voire à des idéologies. Par exemple, des modèles comme celui des lieux centraux, avec ses consommateurs parfaitement informés, dotés de pouvoirs de décision économiquement rationnels, et l'absence de références aux structures oligopolistiques fait assez nettement référence au néo-libéralisme économique, ou plus exactement aux théories de l'économie libérale. Autre exemple, le modèle d'évolution du sousdéveloppement de $Y$. Lacoste fait assez explicitement référence au matérialisme historique, qui est, lui, au moins une théorie, certains diront une idéologie ou une philosophie.

Notion de système enfin. Le système est, rappelons-le, un ensemble d'ensembles (ensemble d'objets munis de leurs attributs, ensemble de relations entre les objets du système, ensemble de relations entre le système et le monde qui lui est extérieur, en amont et en aval, avec les entrées et les sorties).

Beaucoup de modèles sont des modèles de systèmes. Mais centains modèles décrivent une relation, non des ensembles de relations, sans lesquels il n'y a pas vraiment de système. Donc les notions de modèle et de système ne se recouvrent pas. Les modèles de système sont différents des systèmes, puisque tout modèle diffère de la réalité qu'il décrit; tous les systèmes sont modélisables, mais tous les modèles ne sont pas des modèles de systèmes, comme il vient d'être dit.

La notion de modèle comporte une série de connotations qui lui sont souvent liées, mais qui ne font pas partie de la définition :

- le modèle est une abstraction, légitime et indispensable, comme les concepts ;

- les modèles peuvent être formalisés mathématiquement, mais ne le sont pas forcément. A cet égard, il y aurait lieu de mettre à part les modèles mathématiques utilisés dans des démarches qui ne sont pas forcément des élaborations de modèles. Ainsi les analyses factorielles en composante principales utilisent un modèle mathématique (modèle linéaire), et il importe d'en tenir compte. Mais à mon avis, si elles peuvent servir à élaborer des modèles, elles ne sont pas des modèles, mais plutôt des procédures de classement et d'organisation. D'ailleurs, le modèle mathématique est une réalité un peu différente de celle que nous avons définie plus haut ;

- les modèles peuvent être utilisés pour la prévision ;

- les modèles peuvent servir à montrer des analogies structurelles entre des phénomènes très différents, souvent entre des phénomènes simples et bien connus et des phénomènes plus complexes et/ ou moins bien connus. 
En conclusion sur ce point, on soulignera que tout le monde fait plus ou moins usage de modèles, mais souvent sans le dire et parfois sans le savoir. A mon avis, on a tort de se moquer de $M$. Jourdain, de sa joie quand il découvre qu'il faisait de la prose sans le savoir. Connaître le langage dont on se sent permet d'en utiliser correctement la grammaire, donc de tenir un discours cohérent.

\section{II - Fonctionnement des modèles}

On a résumé le "fonctionnement» des modèles dans un schéma (cf. Figure 1). On a indiqué une phase d'élaboration du modèle, qui laisse une part plus ou moins grande à l'observation empirique, puis une phase de test du modèle par confrontation avec la réalité.

- Cette confrontation peut être diachronique : soit les villes turques et leur population à la fin du dix-neuvième siècle ; soit un modèle qui est fondé sur des hypothèses à propos des interactions entre villes qui incitent à établir une voie de chemin de fer. Simulons les voies ferrées dont on peut attendre la construction si le modèle est efficace. Le test consistera alors à confronter les voies existantes à celles que feraient attendre le modèle ;

- La confrontation peut être synchronique. Soit une hypothèse sur l'origine des flux de voyageurs entre ville; et un modèle qui l'exprime. Calculons les flux attendus en fonction du modèle, et confrontons-les aux flux observés. Cette confrontation constitue un test du modèle.

Figure 1: Schéma du fonctionnement des modèles

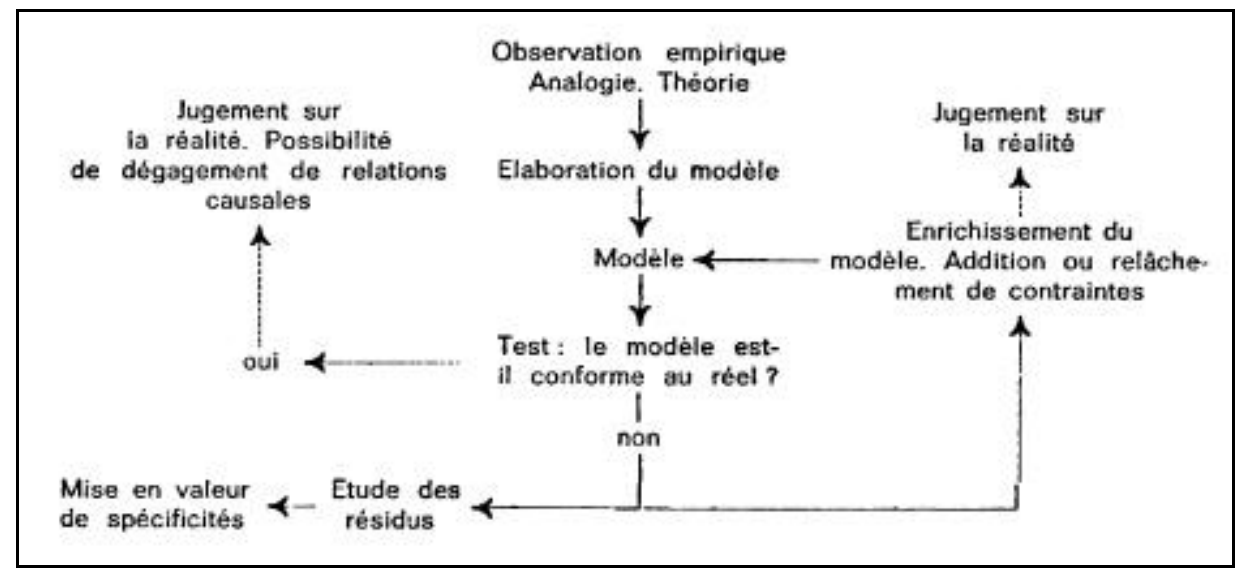

Les flèches pleines indiquent le cheminement logique dans la conception et l'étude des modèles. Les flèches en tireté indiquent les résultats acquis et constituent les " sorties » du cheminement logique. On voit qu'elles sont au nombre de trois, et de nature assez variée. 
Le schéma appelle un certain nombre de commentaires :

- Il montre la double utilité des modèles. Et ceci particulièrement en géographie, avec son souci du spécifique.

- L'intérêt du contenu du modèle, qu'il résulte de la première construction ou des additions successives nécessaires pour le rendre plus utile (cf. la boucle du test). Ces additions successives sont particulièrement significatives. Certains auteurs semblent être partis de modèles volontairement pauvres, avec des contraintes simplificatrices très fortes, qu'ils ont relâchées ensuite une par une ou simultanément (ainsi Von Thünen avec l'espace d'abord homogène du point de vue du coût du transport, puis muni d'axes de transport privilégiés, etc.).

En fait, ce relâchement des contraintes, ou au contraire l'addition de contraintes nouvelles permet une des seules formes d'expérimentation offertes aux géographes.

Le contenu des modèles est varié. Il comporte notamment des éléments, et des relations. Lorsque ces relations sont exprimées par des fonctions reliant des variables, on peut souvent améliorer l'ajustement du modèle à la réalité en changeant la valeur des paramètres dans les équations. $0 r$, cette valeur est souvent significative. Par exemple, dans le modèle de gravité :

$$
I(i, j)=\frac{P i . P j}{(D(i, j))^{n}} 1
$$

La valeur de n exprime une réalité importante, la friction de la distance dans le milieu considéré. La valeur de n est susceptible d'être étudiée, cartographiée, expliquée, etc.

- La confrontation des résultats du modèle et de la réalité donne des différences, des « résidus ». Certes, on peut réduire ceux-ci, mais il est souvent impossible de les éliminer complètement. $D$ 'ailleurs, en géographie particulièrement, ils sont matière à réflexion. Comme l'écrit $P$. Claval, " l'intérêt principal d'une théorie (ne vaudrait-il pas mieux dire modèle ? F. D.-D.) comme celle des lieux centraux ne provient pas de la manière plus ou moins parfaite dont elle rend compte des régularités observables, mais au contraire des problèmes qu'elle pose lorsque ces régularités n'existent pas; elle est génératrice de problèmes, car elle postule un ordre, et tout ce qui ne se conforme pas à cet ondre demande explication, donc enquête » (Rev. Géogr. de l'Est, 1966, p. 147). Même lorsque la confrontation modèle-réalité montre que le modèle en "explique » une partie importante, il est intéressant de réfléchir sur le reste, qui exprime souvent la spécificité de l'espace considéré, ou de ses différentes parties, car les résidus peuvent être inégalement répartis, donc susceptibles de représentation cartographique. Il est souvent intéressant d'élaborer un modèle "faible », comportant peu d'hypothèses, mais qui exprime ce que la réalité a de plus banal (au sens de P. Birot, quand il parle de « formes banales »). L'écart au banal montre alors bien les parties du réel que celui-ci n'explique pas, qui ménitent donc une explication complémentaire.

Les exemples de démarches de ce genre sont multiples. Ainsi, dans la simulation des réseaux hydrographiques, la fixation de quelques règles simples et des tirages

\footnotetext{
1 I(i,j) est une mesure du degré d'interaction entre les éléments i et j, Pi et Pj une mesure de la masse de ces éléments et $\mathrm{D}(\mathrm{i}, \mathrm{j})$ la distance qui les sépare. Dans le cas de deux villes, par exemple, $\mathrm{Pi}$ et $\mathrm{Pj}$ peuvent être leur population, ou les effectifs d'une catégorie sosio-prefessionnelle, etc.
} 
au hasard permettent d'obtenir des réseaux dendritiques, qui montrent le rôle des processus banaux. Mais ces formes simulées diffèrent de la réalité dans le cas où jouent des influences qui n'ont pas été modélisées, comme celle de la structure par exemple.

De même, une carte de la fréquentation de lieux de pèleninage Jaïn du Gujrat ne montre à l'inspection visuelle rapide qu'une influence assez banale, celle de la distance. Si cette influence est modélisée, et qu'ensuite le modèle est confronté à la réalité, on obtient une carte des résidus qui montre une contribution très différente des communautés jaïns au pèleninage : différences qui doivent être expliquées, et relancent l'enquête ; dans le cas précis, celle-ci a abouti à des résultats intéressants sur les degrés variés de l'orthodoxie de quelques sectes jaïns...

A la limite, le modèle peut jouer un rôle comparable à celui des hypothèses nulles qu'on fait intervenir dans les tests de signification.

- Le schéma permet aussi de montrer que la variété des modèles provient de différences qui se manifestent à deux niveaux. Il est en effet courant de classer les modèles en fonction de leur degré d'abstraction croissante. Mais il arrive qu'on mêle en réalité deux ordres de faits différents : le degré d'abstraction du langage utilisé par le modèle d'une part, et la place respective de l'observation empirique et du " raisonnement théorique » dans la conception du modèle de l'autre. 0 n parle parfois de " modèle abstrait " sans qu'on sache si l'on veut dire par là qu'il est exprimé dans un langage abstrait, mathématique par exemple, ou qu'il est largement fondé sur une analogie théorique ou un raisonnement a prioni.

Il semblerait donc préférable de bien distinguer les deux critères, et pas seulement pour la satisfaction de présenter une classification plus logique. L'utilisation qu'on fera légitimement d'un modèle dépend en effet de ses caractères, de ses propriétés situées en fonction de celles des autres modèles. Autant vaut s'en faire une idée précise.

C'est ce qui nous amène à présenter le schéma 2, qui montre comment ces modèles se situent sur deux axes, l'un exprimant l'abstraction croissante du langage utilisé dans le modèle lui-même, l'autre le passage des modèles qui sont une simple mise en forme de la réalité empirique, à ceux qui sont dus pour l'essentiel à des raisonnements ou des analogies, à la déduction.

Langage et mode de conception peuvent être liés : les modèle les plus concrets, comme les modèles iconiques ou analogue (cartes et " modèles réduits ») doivent évidemment beaucoup à l'observation directe de la réalité empirique. Inversement, le modèle de gravité, fondé sur l'analogie, a une formalisation essentiellement mathématique. Mais cette correspondance langage/ mode de conception est loin d'être un fait général.

Un «modèle réduit» (langage très concret) peut avoir été conçu en fonction d'hypothèses très théoriques. Par exemple, on supposera que l'atmosphère peut être traitée comme un fluide incompressible ou le glacier comme un plastique parfait, et l'on construira de modèles réduits pour tenter de simuler quelques caractères de la circulation atmosphérique ou de la vallée glaciaire en utilisant des liquides au lieu d'air ou des solides plastiques au lieu de glace.

Inversement, l'idée de l'existence d'une loi linéaire dans la relation entre deux phénomènes peut se dégager d'observations simples mais sa formalisation est évidemment une fonction mathématique donc abstraite.

${ }^{2}$ Comme par exemple les maquettes de ports. 
Figure 2 : Classement des modèles en fonction de deux critères

\begin{tabular}{|c|c|c|c|c|}
\hline \multirow{2}{*}{$\begin{array}{l}\text { Critère : } \\
\text { abstraction } \\
\text { croissante du mode } \\
\text { de conception } \\
\text { du modèle }\end{array}$} & \multicolumn{4}{|c|}{ Critère : abstraction croissante de la forme du modèle } \\
\hline & Images & $\begin{array}{c}\text { Modèles physiques } \\
\text { concrets }\end{array}$ & Simulation & Mathématiques \\
\hline $\begin{array}{l}\text { Modèles } \\
\text { empiriques }\end{array}$ & & Modèle réduit & $\begin{array}{l}\text { Modèles de } \\
\text { diffusion }\end{array}$ & $\begin{array}{l}\text { Régression } \\
\text { linéaire }\end{array}$ \\
\hline Systèmes & $\begin{array}{l}\text { Schéma-image } \\
\text { de système }\end{array}$ & & & $\begin{array}{l}\text { Fomalisation } \\
\text { mathématique } \\
\text { d'un système }\end{array}$ \\
\hline $\begin{array}{l}\text { Modèles } \\
\text { analogiques }\end{array}$ & & $\begin{array}{l}\text { Modèle simulant } \\
\text { la circulation } \\
\text { atmosphérique à } \\
\text { l'aide de liquides }\end{array}$ & & $\begin{array}{c}\text { Modèle de } \\
\text { gravité } \\
\text { Modèle de } \\
\text { Von thünen }\end{array}$ \\
\hline $\begin{array}{l}\text { Modèles } \\
\text { théoriques }\end{array}$ & & & & \\
\hline
\end{tabular}

N.B. Les modèles de simulation sont généralement considérés comme moins abstraits que les modèles mathématiques. Ils utilisent en effet un langage mathématique, mais surtout des probabilités, que l'on fait jouer dans le cadre de règles fixées à partir de l'observation empirique.

Ce schéma ne prétend nullement être exhaustif. Dans le tableau ne figurent que quelques exemples de modèles, situés les uns par rapport aux autres. Nous n'avons cherché qu'à présenter une méthode de classement, et non une classification complète.

D'un autre point de vue, on notera que des modèles semblables par leur mode de conception peuvent être formalisés de façons très différentes. Ainsi, les modèles de systèmes. Ils font appel à une combinaison de l'observation empirique et du raisonnement prioni, qui les situe en position moyenne sur l'axe vertical de la Figure 2. Ils peuvent être exprimés soit par un discours verbal, soit par un schéma, soit par un ensemble d'équations. Chaque type de langage a son intérêt. Nous avons fait une expérience assez significative en essayant de schématiser la conception que se faisait M. Sorre d'un certain type de système de cultures, en partant d'un texte de cet auteur. Alors que le discours verbal permettait de parler d'un système unique, la rigueur des définitions imposée par la schématisation nous a obligé à décomposer le système de culture en deux sous-systèmes, comme le montre la Figure 3. On ressent ici une nécessité de précision et de rigueur un peu analogue à celle que l'on rencontre lors de l'élaboration de la légende d'une carte : tous les géographes savent comment cela les oblige à préciser leurs concepts. On peut penser qu'un usage de modèles, même formalisés par de simples schémas, pourrait également leur faire gagner beaucoup de précision. 
Figure 3 : Essai de schématisation d'un système de cultures (l'assolement triennal européen)

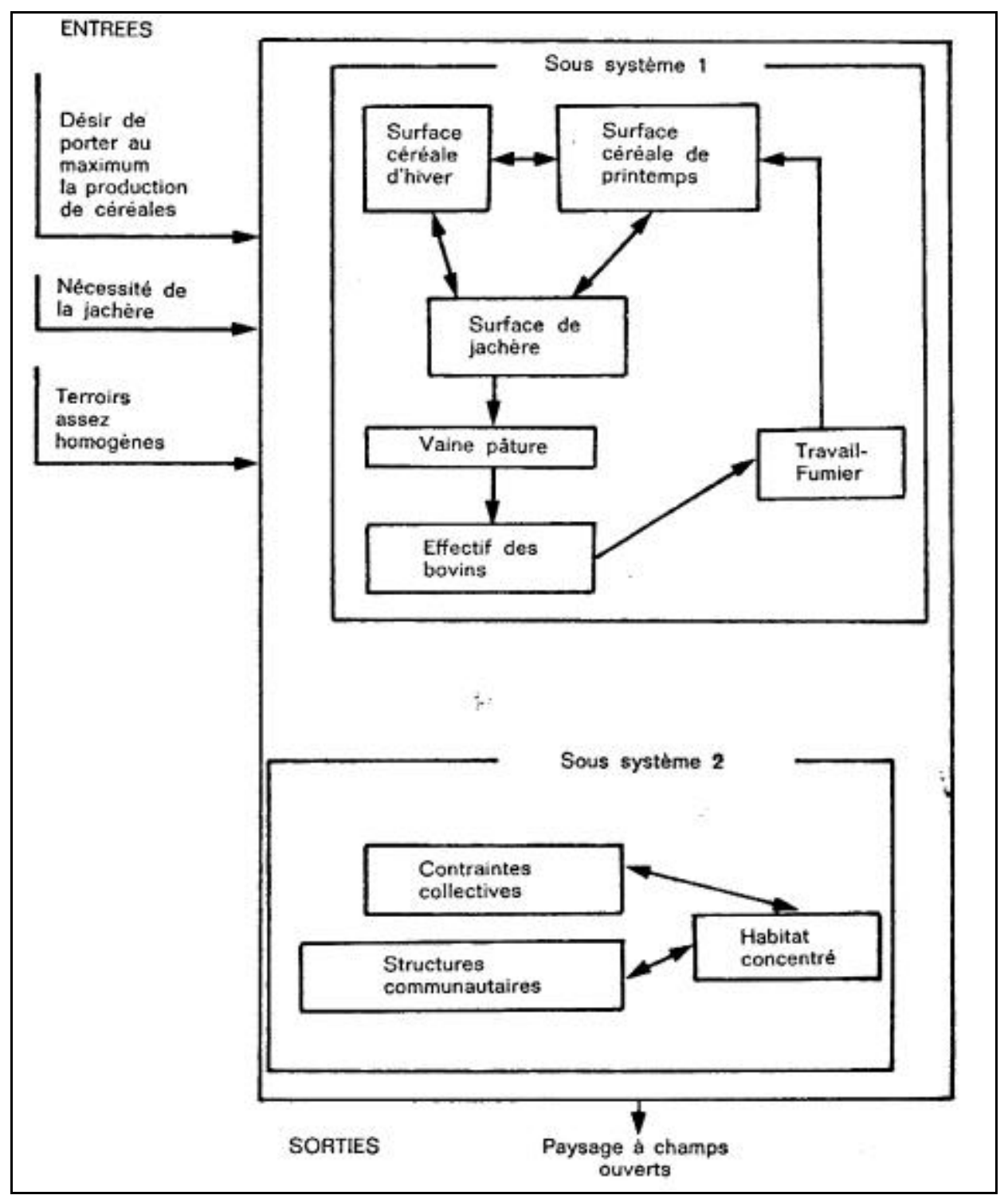

La signification des flèches n'est pas la même dans les deux sous-systèmes. La contrainte exprimée est plus forte dans le sous-système 1 que dans le sous-système 2.

La figure est extraite d'une étude sur les structures et les systèmes en géographie, par 0. DoLlfus et F. D URAND-DASTES, à paraître prochainement ("Some Remarks on the Notions of Structure and System in Geography ", 1975, Geoforum, vol. 6, n², p. 83-94).

Bulletin de l’Association de Géographes Français, 1974, n 413-414, p. 43-50. 